\title{
Anion Separation by Selective Crystallization of Metal-Organic Frameworks
}

\author{
Radu Custelcean*, Tamara J. Haverlock, and Bruce A. Moyer
}

Chemical Sciences Division, Oak Ridge National Laboratory, Oak Ridge, Tennessee 37831-6119, USA

custelceanr@ornl.gov

\section{Supporting Information}




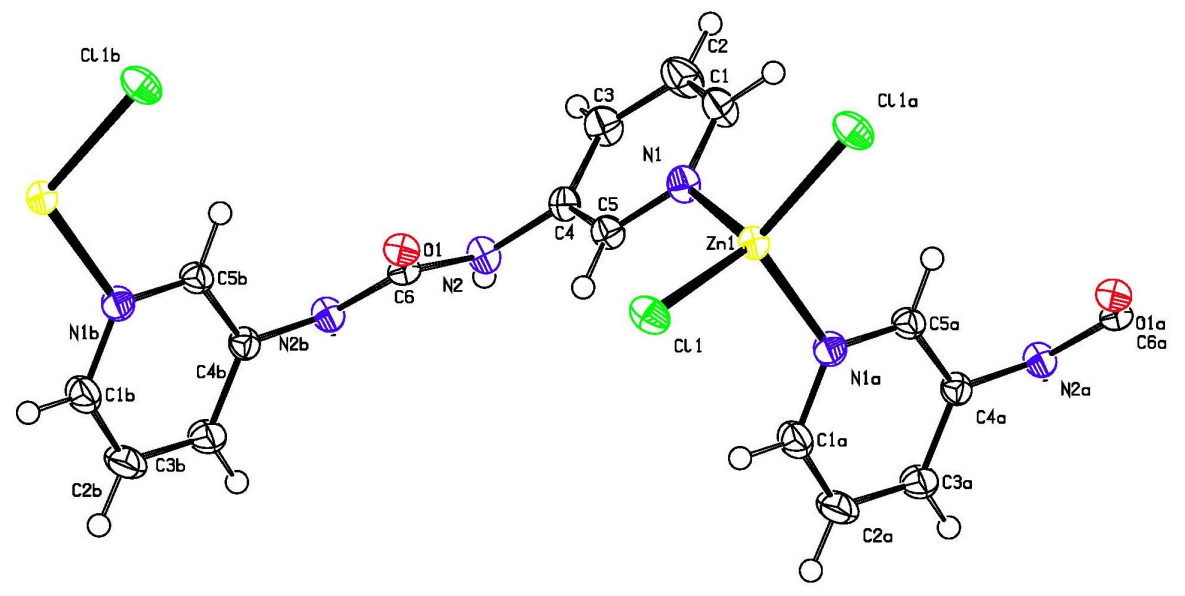

Figure S1. Crystal structure of 1, showing the atoms as thermal ellipsoids at the $50 \%$ probability level.

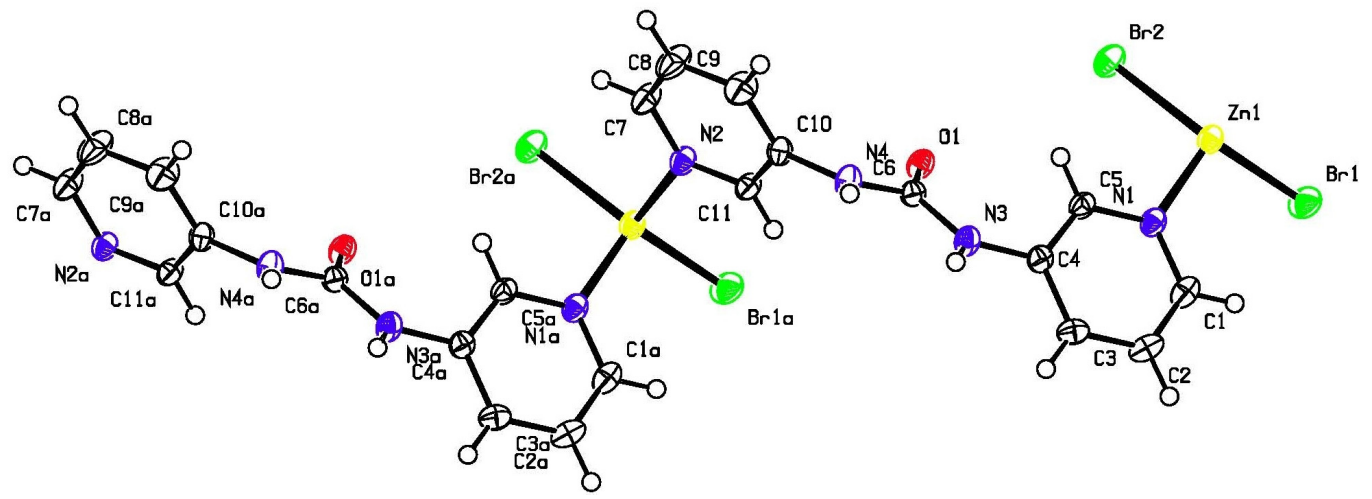

Figure S2. Crystal structure of 2, showing the atoms as thermal ellipsoids at the $50 \%$ probability level.

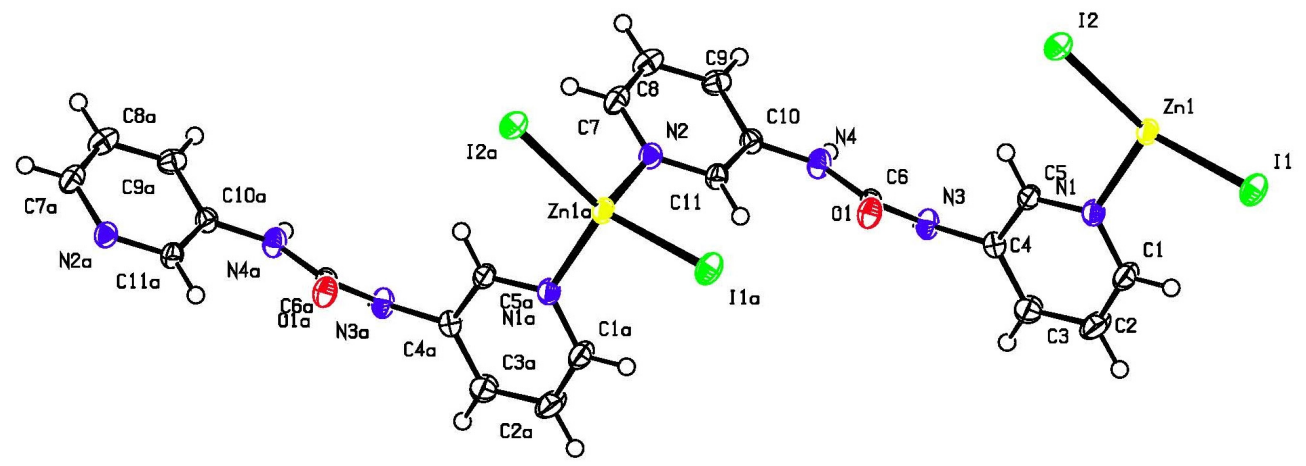

Figure S3. Crystal structure of $\mathbf{3}$, showing the atoms as thermal ellipsoids at the $50 \%$ probability level. 


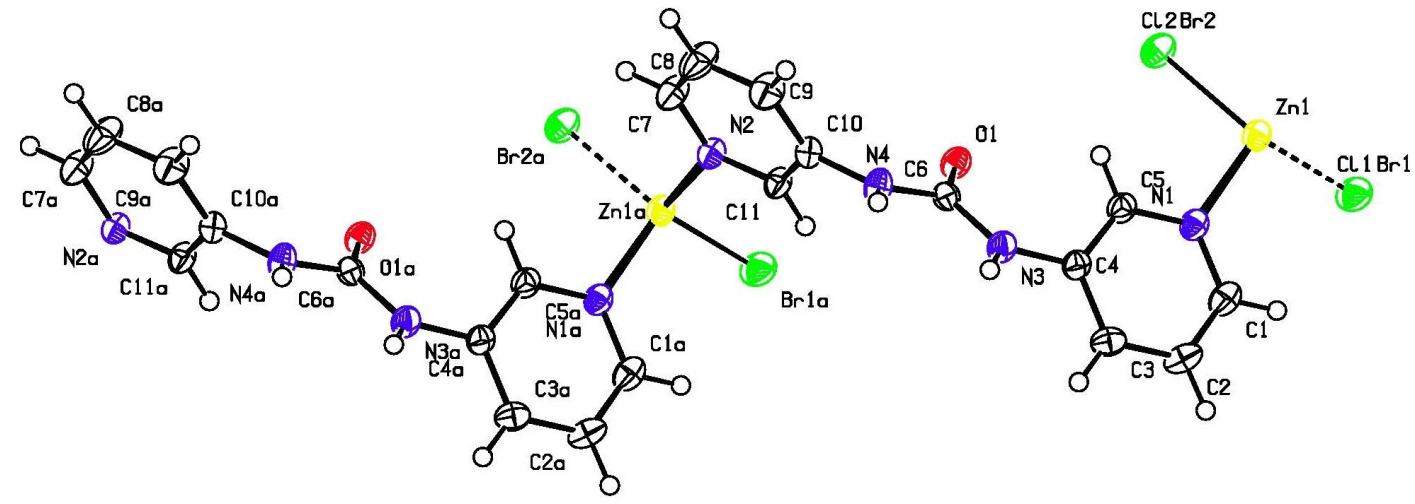

Figure S4. Crystal structure of 4, showing the atoms as thermal ellipsoids at the $50 \%$ probability level.

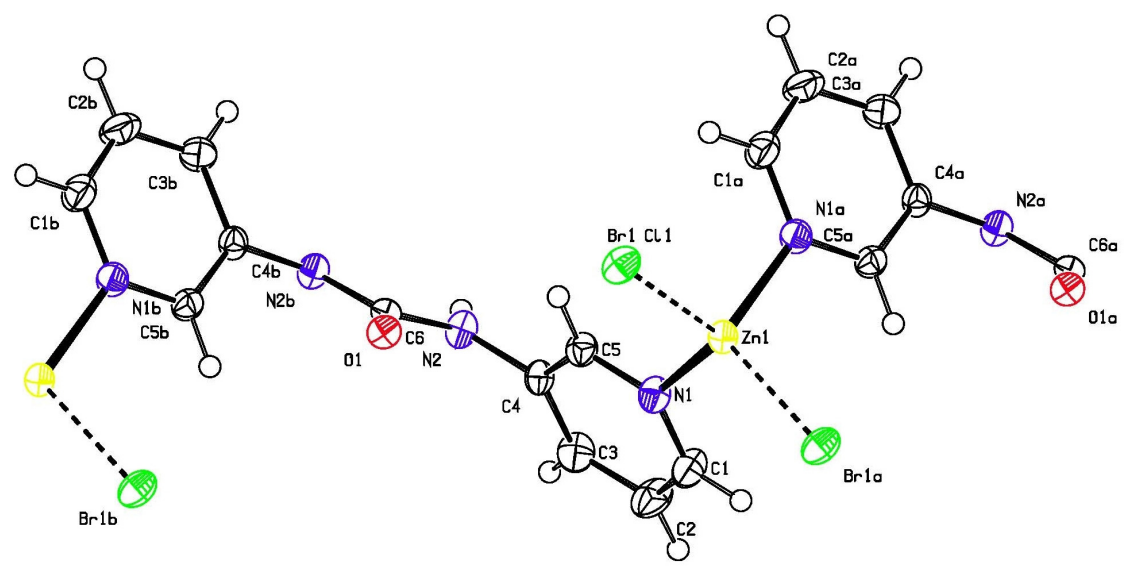

Figure S5. Crystal structure of 5, showing the atoms as thermal ellipsoids at the $50 \%$ probability level.

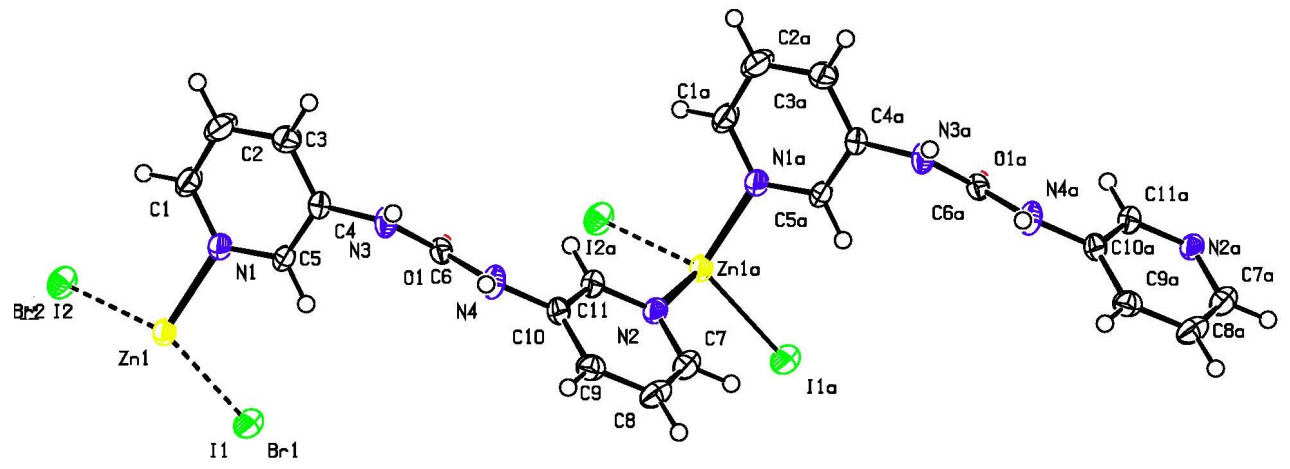

Figure S6. Crystal structure of 6, showing the atoms as thermal ellipsoids at the $50 \%$ probability level. 


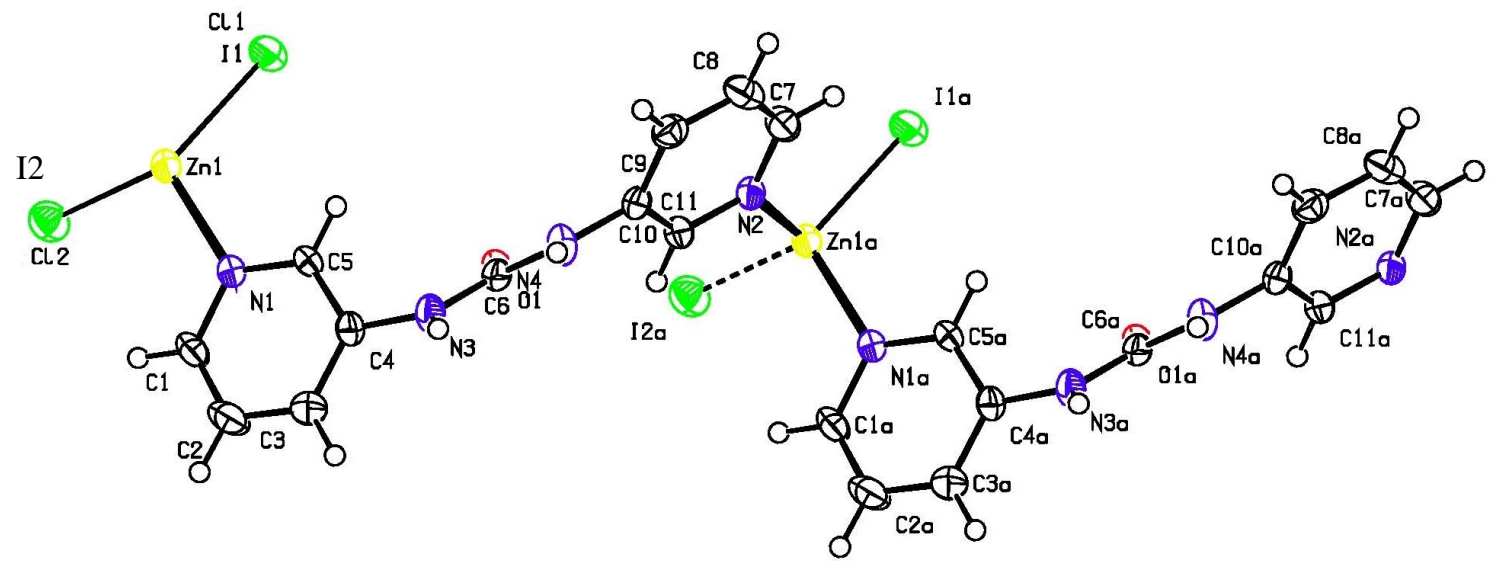

Figure S7. Crystal structure of 7, showing the atoms as thermal ellipsoids at the $50 \%$ probability level. 